\title{
Editorial
}

\section{Bioimpedance Sensors: Instrumentation, Models, and Applications}

\author{
David Naranjo-Hernández $\mathbb{D},{ }^{1}$ Javier Reina-Tosina $\mathbb{D},{ }^{1}$ \\ Rubén Buendía $\left(\mathbb{1},{ }^{2}\right.$ and Mart Min $\left(\mathbb{1}^{3}\right.$ \\ ${ }^{1}$ University of Seville, Seville, Spain \\ ${ }^{2}$ University of Borås, Borås, Sweden \\ ${ }^{3}$ Tallinn University of Technology, Tallinn, Estonia
}

Correspondence should be addressed to David Naranjo-Hernández; dnaranjo@us.es

Received 13 June 2019; Accepted 18 June 2019; Published 16 July 2019

Copyright (C) 2019 David Naranjo-Hernández et al. This is an open access article distributed under the Creative Commons Attribution License, which permits unrestricted use, distribution, and reproduction in any medium, provided the original work is properly cited.

Population aging and the consequent increase in chronic patients are driving research and development of multiple biomedical sensor technologies. These allow for better monitoring of people's health status as well as improved diagnosis, which anticipate a more personalized, preventive, and proactive healthcare. Bioimpedance technology offers valuable information about tissue/cell physiology and pathology. This way bioimpedance sensors technology is becoming the basis of novel and noninvasive medical diagnostic devices.

Considering the frequency response of bioimpedance and the dielectric/conductive properties of the different tissues it is possible to estimate the diverse compartments of the human body, i.e., intracellular and extracellular water, lean mass, fat mass, etc. The clinical usefulness of body composition analysis by bioimpedance techniques (BIA) has been demonstrated in multiple clinical areas: nephrology, for the optimal management of renal patient excess fluid; nutrition, for a better control of the patient's nutritional status; also during pregnancy and lactation, as a marker or direct cause of diseases; during the decision-making process about a disease, as a complement to the diagnosis and monitoring of conditions related to the cardiovascular system or in oncology, among others. Body composition analysis is also useful in chronic diseases, such as chronic obstructive pulmonary disease, where the loss of body weight and the decrease in muscle mass are risk factors associated with increased morbidity and mortality and deterioration in the quality of life.

Changes in fluids and volumes in the thoracic cavity as result of cardiac hemodynamics also produce changes in the thoracic impedance which allow estimating parameters related to the mechanical function of the heart such as cardiac output, stroke volume, systolic time ratio, and the time of ejection of the left ventricle or the preejection period. The application of bioimpedance technology in the monitoring of cardiac parameters is called impedance cardiography (ICG), which has been proven very useful in the diagnosis of cardiovascular diseases as well as in trauma emergencies.

Bioimpedance measurements allow for the characterization of biological media and organic tissues and have been used for detection of edema, diagnosis of skin-related diseases, detection of cancerous tissues, and monitoring of ischemia during the transplant process and even as a noninvasive and nonobstructive estimation of blood pressure and blood glucose level. Bioimpedance techniques are also the basis of some laboratory devices for the monitoring of cell cultures and the study of the mechanical properties of cells (population, motility, viability, etc.).

The application of bioimpedance to obtain medical images of the inside of the human body using the technique known as electrical impedance tomography (EIT) is as well noteworthy. Despite the low resolution of the images 
obtained, EIT has other advantages. It is particularly useful for monitoring lung function because lung tissue resistivity is five times higher than most other soft tissues in the thorax.

Despite the enormous potential of bioimpedance technology, its application to the clinical practice is still limited and a significant research effort is required to address the future challenges: electrodes for continuous monitoring, miniaturization of bioimpedance devices, reduction of energy consumption, improvement of sensitivity and specificity, detection and correction of artifacts, and application in implants, biosensors, and Lab-on-Chip technologies. This special issue is aimed at surveying recent advances in bioimpedance sensors, devices, and applications. Out of sixteen submissions, five articles were accepted after a thorough and rigorous peerreview process. A brief introduction of the published articles follows.

The paper "Orthogonal Multitone Electrical Impedance Spectroscopy (OMEIS) for the Study of Fibrosis Induced by Active Cardiac Implants" by E. De Roux et al. is devoted to the development and implementation of the simultaneous multitone impedance spectroscopy for detection as well as characterization of tissue modifications (fibrosis) around the implanted electrodes. Such electrodes are in use for both, stimulation and sensing, in implantable cardiac rhythm management devices (pacemakers and defibrillators), including also the data acquisition in implantable cardiac monitors. Because the fibrosis reduces susceptibility to stimulation (pacing) and sensibility for sensing, the methods for early detection of fibrosis are of great importance.

The paper "Electrical Impedance-Based Methodology for Locating Carcinoma Emulators on Breast Models" by $\mathrm{M}$. Gutierrez-Lopez et al. describes the Anomaly Tracking Circle algorithm. This is a novel method to estimate the presence and location of breast carcinoma based on bioimpedance measurements through eight $\mathrm{Ag} / \mathrm{AgCl}$ electrodes uniformly distributed in a ring configuration. Whereas all different technologies for breast tumor diagnosis have pros and cons, bioimpedance promises a painless, minimally invasive diagnosis at a low cost. Despite the method being in an early stage (it was evaluated on experimental agar breast models), results show great promise.

A key issue for bioimpedance-based measurement systems is the electrode configuration and material. In the paper "The Influence of Blood Glucose Meter Resistance Variation on the Performance of a Biosensor with a Gold-Coated Circuit Board" by K.-Y. Kim et al. the authors present a novel test strip for blood glucose measurement. Their contribution is based on the analysis of the effects of glucose oxidase in the reaction zone of gold-coated test strips, which provides a way of tuning the anodic and reductive peaks, thereby adjusting the resistance of the electrochemical reaction. To validate their approach, an experimental study was conducted in which glucose oxidase was combined with standard solutions with different concentrations and the corresponding current changes were measured. Results reveal that the accuracy of blood glucose measurement can be influenced by adjusting the resistance of the electrode test strip.

The work entitled "Simultaneous Recording of ICG and ECG Using Z-RPI Device with Minimum Number of
Electrodes" by A. Hadif et al. describes a device and a procedure to monitor ICG and electrocardiogram (ECG) signals simultaneously using the same electrodes for both measurements, as opposed to the common methodology that uses separate electrode configurations. The results presented show the viability of the proposed scheme, constituting a step forward to the development of portable biomedical sensor technologies.

Last but not least, the paper titled "Fundamentals, Recent Advances, and Future Challenges in Bioimpedance Devices for Healthcare Applications" by D. Naranjo-Hernández et al. performs a complete review of bioimpedance technology and its applications in biomedical sensors. A strength of this study is that it provides the reader with a complete view of the technology, from the physical and physiological basis of bioimpedance, through the different mathematical models proposed in the literature, to the main instrumentation schemes. The main problems of bioimpedance measurements are also analyzed, such as the electrode issues or the noise and artifacts. Since bioimpedance is a very broad and heterogeneous concept, the fundamentals and results of the main applications of bioimpedance are shown in a structured way in separate sections: BIA, ICG, IET, transthoracic impedance pneumography, skin conductance, and biosensors. A final section analyzes recent advances and challenges and future applications of bioimpedance.

It is exciting to present this special issue on recent advances in bioimpedance technology and we hope that the articles published will promote discussions and inspire new innovations towards the improvement of people's health and quality of life.

\section{Conflicts of Interest}

The editors declare that they have no conflicts of interest regarding the publication of this special issue.

\section{Acknowledgments}

We would like to thank all the authors for their excellent contributions and also the reviewers for their valuable help and constructive criticism.

David Naranjo-Hernández Javier Reina-Tosina Rubén Buendía Mart Min 


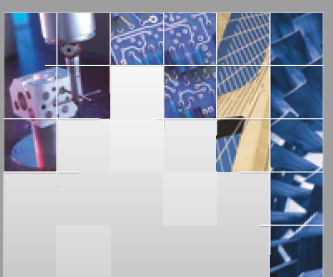

\section{Enfincering}
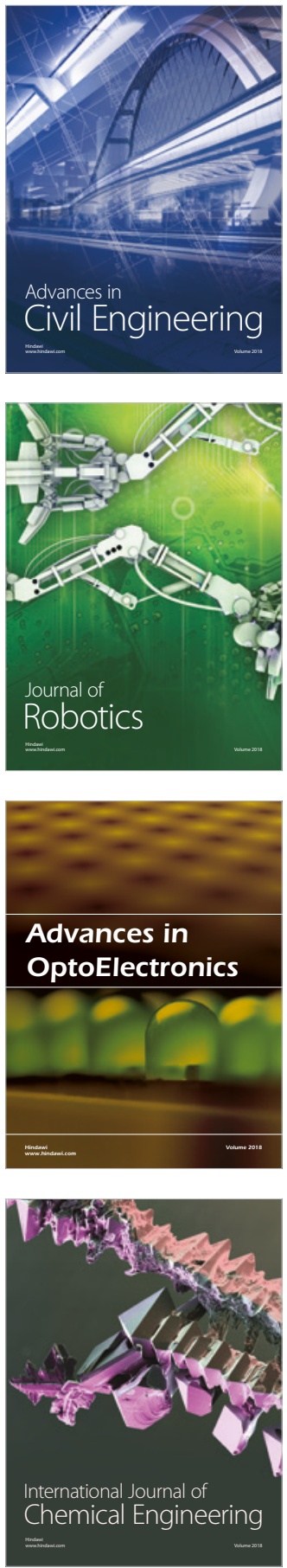

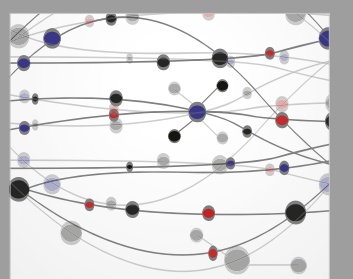

\section{Rotating \\ Machinery}

The Scientific World Journal

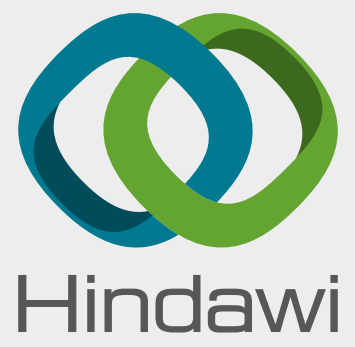

Submit your manuscripts at

www.hindawi.com
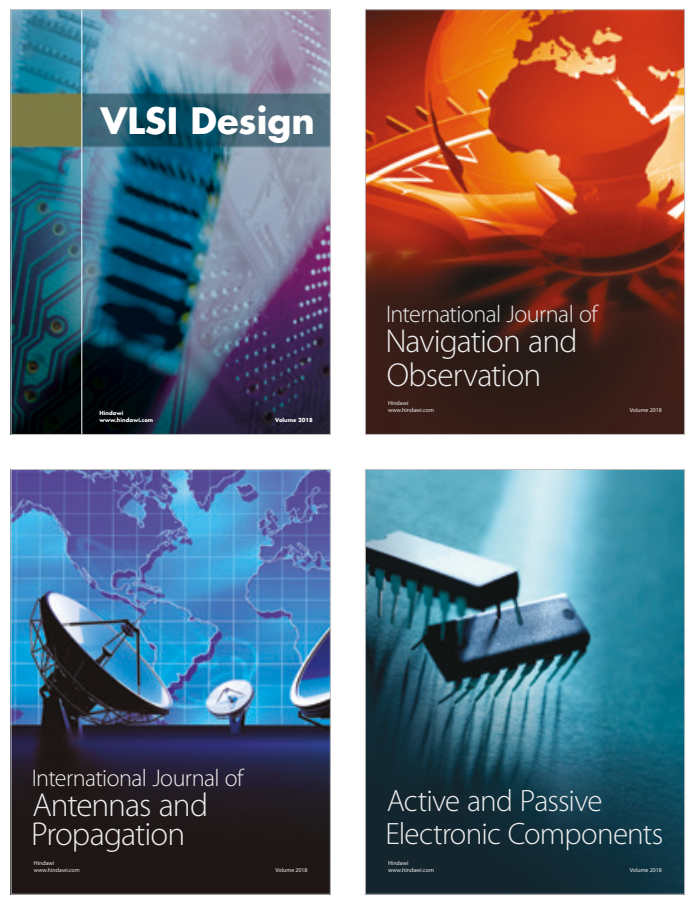
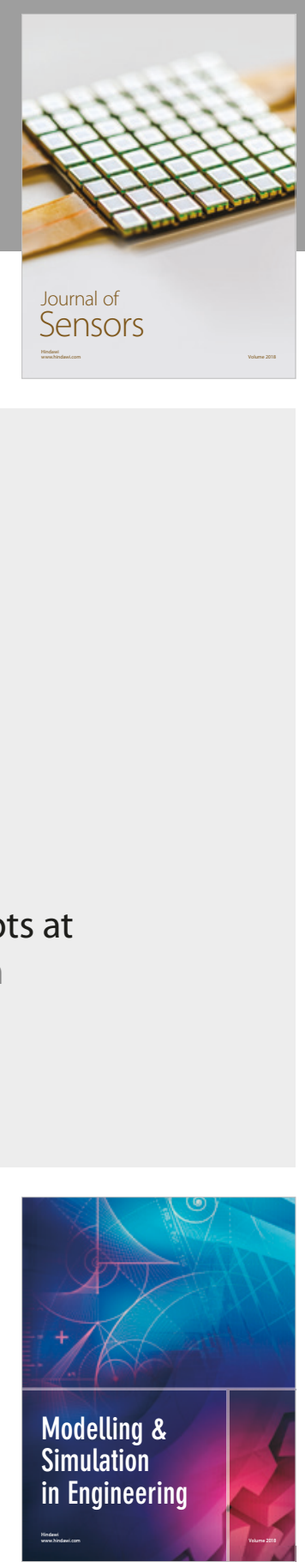

\section{Advances \\ Multimedia}
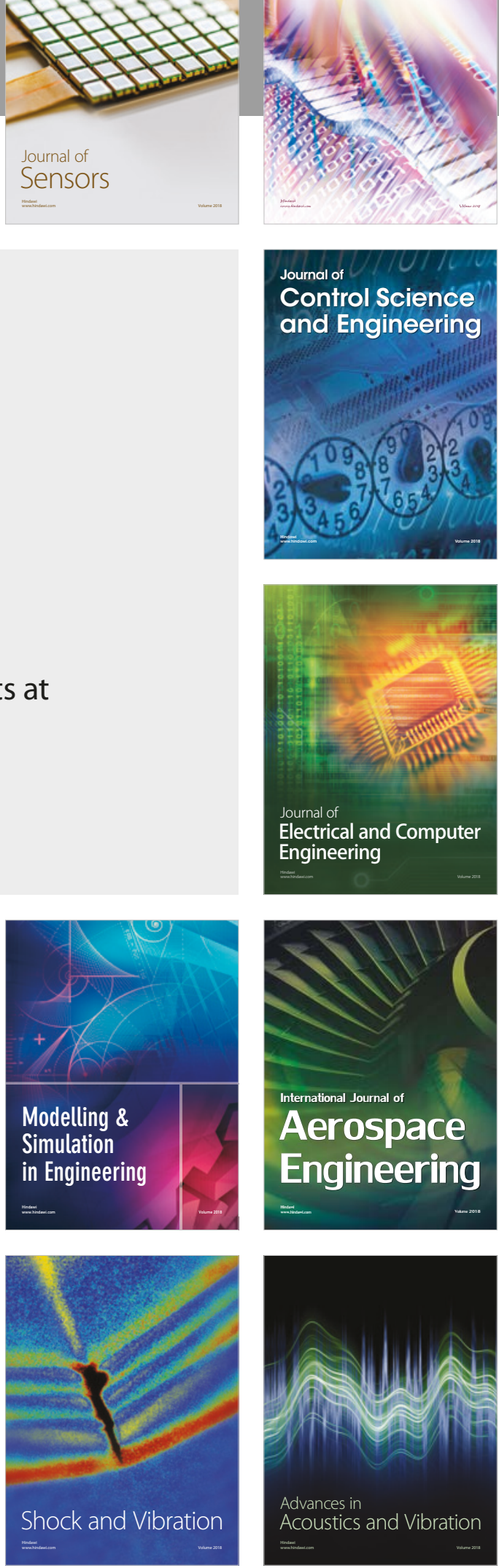